\title{
A Consolidated Visualization of Wind Farm Energy Production Potential and Optimal Land Shapes under Different Land Area and Nameplate Capacity Decisions
}

\author{
Weiyang Tong* \\ Syracuse University, Syracuse, NY, 13244 \\ Souma Chowdhury ${ }^{\dagger}$ and Achille Messac ${ }^{\ddagger}$ \\ Mississippi State University, Mississippi State, MS, 39762
}

\begin{abstract}
Effective and time-efficient decision-making in the early stages of wind farm planning can lay the foundation of a successful wind energy project. Undesirable concept-to-installation delays in wind farm development is often caused by conflicting decisions from the major parties involved (e.g., developer, investors, landowners, and local communities), which in turn can be (in a major part) attributed to the lack of an upfront understanding of the trade-offs between the technical, socio-economic, and environmental-impact aspects of the wind farm for the given site. This paper proposes a consolidated visualization platform for wind farm planning, which could facilitate informed and co-operative decision-making by the parties involved. This visualization platform offers a GUI-based land shape chart, which provides the following information: the variation of the energy production capacity and of the corresponding required optimal land shape with different land area and nameplate capacity decisions. In order to develop this chart, a bi-objective optimization problem is formulated (using the Unrestricted Wind Farm Layout Optimization framework) to maximize the capacity factor and minimize the land usage, subject to different nameplate capacity decisions. The application of an Optimal Layout-based land usage estimate allows the wind farm layout optimization to run without pre-specifying any farm boundaries; the optimal land shape is instead determined as a post process, using convex hull and minimum bounding rectangle concept, based on the optimal arrangement of turbines. Three land shape charts are generated under three characteristic wind patterns - (i) single dominant wind direction, (ii) two opposite dominant wind directions, and (ii) two orthogonal dominant wind directions, all three patterns comprising the same wind speed distribution. The results indicate that the optimal land shape is highly sensitive to the variation in LAMI for small-capacity wind farms (few turbines) and to the variation in nameplate capacity for small allowed land area. For the same decided nameplate capacity and LAMI values, we observe reasonable similarity in the optimal land shapes and the maximum energy production potentials given the "single dominant direction" and the "two opposite dominant directions" wind patterns; the optimal land shapes and the maximum energy production potentials yielded by the "two orthogonal dominant directions" wind pattern is however observed to be relatively different from the other two cases.
\end{abstract}

Keywords: Decision making, Land Area per MW Installed, Land shape chart, Optimal Layout-based land use, Smallest Bounding Rectangle, Visualization platform

\footnotetext{
*Doctoral student, Multidisciplinary Design and Optimization Laboratory, Department of Mechanical and Aerospace Engineering. AIAA Student Member.

${ }^{\dagger}$ Research Assistance Professor, Department of Mechanical Engineering, Center for Advanced Vehicular Systems. AIAA Senior Member.

${ }^{\ddagger}$ Dean of Engineering and Professor, Earnest W. and Mary Ann Deavenport Jr. Chair, Bagley College of Engineering. AIAA Lifetime Fellow. Email: messac@bagley.msstate.edu

Copyright (c) 2013 by Achille Messac. Published by the American Institute of Aeronautics and Astronautics, Inc. with permission.
} 


\section{Introduction}

The development of wind farms involves a complex planning process that can be divided into several stages from conceiving the project to installation of turbines. Effective and time-efficient decision-making in the early stages of wind farm planning can lay the foundation for a successful wind farm project. Generally, various important factors are to be determined at this stage, including site selection, wind measurement, negotiation with landowners, and feasibility analysis (e.g., permitting and transmission access). ${ }^{1,2}$ More importantly, most of those factors are inter dependent. Hence, wind farm developers need to coordinate these input factors to seek a balance between engineering, socia-economic, and environmental aspects of the project. However, owing to the lack of transparency governing this development process, obstacles are added causing both technical and socia-economic issues that delay the planning process. ${ }^{3-5}$ Technical issues may include compliance with spatial planning, insufficient grid capacity and land usage, and a lack of planning for grid extension and reinforcements. ${ }^{5-7}$ Socia-economic issues are primarily related to public involvement. In fact, public opposition to wind power development has remained a significant obstacle. ${ }^{8}$ Both the technical and socia-economic issues may result in concept-to-installation delays of a wind energy project, which further impact the economic feasibility of that project as well as that of the overall wind energy industry.

To avoid such delays, effective wind farm planning should allow for cooperative decision making among the major stakeholders, which include wind farm developers, power utilities, project investors, landowners, local public authorities, and local communities, as shown in 1. Unfortunately, in the current wind farm design paradigm, there hardly exists any decision making platform that provides a transparent and consolidated illustration of the technical, economic, and environmental feasibility of the project. A visualization platform is developed in this paper to enable such an informed and co-operative decision-making process. The factors and objectives exhibited in this visualization platform are: (i) wind farm capacity factor decisions, (ii) Land Area per MW Installed (LAMI) decisions, and (iii) nameplate capacity decisions, and their impact on the optimal land shape and the maximum energy production capacity. It is important to note that such a

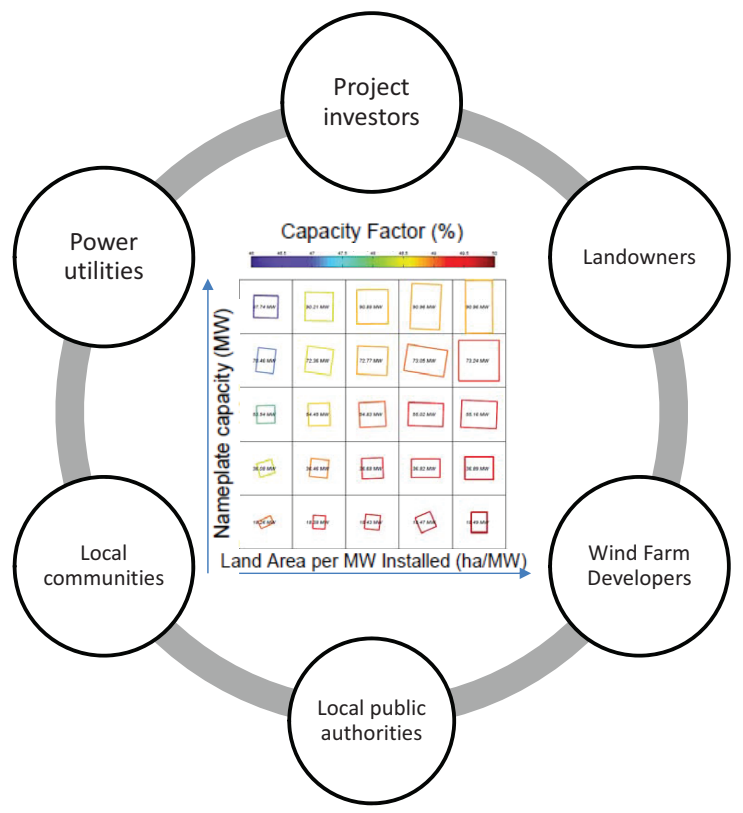

Figure 1. Providing stakeholders a visualization of the impact of total capacity and land usage on the optimal land area that need to be used and on the resulting energy production capacity

visualization platform necessitates hundreds of wind farm layout optimizations that account for the coupled impact of various natural (resource-related) and design factors. The abilities of the Unrestricted Wind Farm Layout Optimization (UWFLO), specifically "computational efficiency" and "coupled impact consideration " are uniquely suited to develop this visualization platform. 


\section{A. Wind Farm Land Usage}

Land usage is an important consideration in wind farm planning. From the design perspective, land usage can be represented in terms of land area and land shape. The design of a wind farm layout is generally optimized to reduce the wake losses and maximize the energy production. However, the maximum energy that can be extracted from a certain farm land shape (defined by specific boundaries) could significantly differ from that given by another farm land shape. On the other hand, large utility-scale wind energy projects demand greater land area for wind farms, since the reduction of wake losses necessitates larger inter-turbine spacing. Therefore, the energy production is strongly related to the allowed land use. ${ }^{9}$

In the case of conventional wind farm layout optimization, land area and land shape are fixed, so the optimal farm layout(s) and the corresponding maximum energy production that can be obtained for a wind resource depend on the given/specified boundaries of the farm site. Farm sites generally stretch over a substantial area, and the boundaries governing turbine installations are themselves subject to planning and need not be fixed. Additionally, large utility-scale wind energy projects are more likely to have concerns regarding permitting framework, the net impact on surroundings (e.g., noise impact and impact on local wildlife), and potential landowner participation related issues. ${ }^{10,11}$ Owing to those concerns, wind energy projects in turn demand minimization of the land area footprint of wind farms, assuming that minimizing the land use will reduce the degree of impact of the wind farm on its immediate surroundings. To explore the trade-off between the maximum energy production and the land usage, in this paper we perform a bi-objective wind farm layout optimization without specifying farm boundaries. Since various nameplate capacities are also considered, we use the wind farm Capacity Factor (CF) and the Land Area per MW Installed (LAMI) to represent the maximum energy production capacity and the land usage, respectively.

It is noted that the land orientation is also an important factor determining the farm layout and affecting the maximum energy production. Chowdhury et al. ${ }^{12,13}$ illustrated the importance of land usage in wind farm planning, where they characterized the influence of land area, land shape (rectangular and circular), and land orientation on the optimal wind farm performance.

\section{B. Wind Farm Energy Production}

The prediction of Annual Energy Production (AEP) is based on the annual distribution of wind conditions (wind speed and wind direction) at the concerned farm site. In this paper, the AEP is determined by integrating the power generation over a wind distribution expressed in terms of $N_{P}$ wind conditions. Assuming the wind farm operates continuously throughout the year (all $365 \times 24$ hours), the AEP of a wind farm, $E_{\text {farm }}$ (in $k W h / y r$ ), can be expressed as

$$
\text { where } \begin{aligned}
E_{\text {farm }} & =(365 \times 24) \sum_{j=1}^{N_{P}} P_{\text {farm }}\left(U_{j}, \theta_{j}\right) f\left(U_{j}, \theta_{j}\right) \Delta U \Delta \theta, \\
\Delta U \Delta \theta & =U_{\text {max }} \times 360^{\circ} / N_{P} \\
P_{\text {farm }}\left(U_{j}, \theta_{j}\right) & =\sum_{k=1}^{N} P_{k}\left(U_{j}, \theta_{j}\right)
\end{aligned}
$$

Here, $U_{\max }$ represents the maximum possible wind speed in the current wind distribution; $f\left(U_{j}, \theta_{j}\right)$ represents the probability of the occurrence of a wind condition defined by incoming wind speed $U$ and wind direction $\theta$; and $P_{\text {farm }}$ is the total power generated by $N$ turbines for an incoming wind speed $U$ from direction $\theta$. The power generated by Turbine- $k$ is represented by $P_{k}$. The power generation model incorporated in the Unrestricted Wind Farm Layout Optimization (UWFLO) framework is used in this paper. ${ }^{14}$ This model quantifies the wind farm power generation as a function of the incoming wind conditions, farm layout, and turbine features. Additionally, it also accounts for the wake merging and variable induction factor.

The wind farm Capacity Factor (CF) can be expressed as

$$
C F=\frac{E_{\text {farm }}}{(365 \times 24) \sum_{k=1}^{N} P_{r k}}
$$

where $P_{r k}$ represents the rated power of Turbine- $k$. 


\section{Development of Wind Farm Cooperative Decision Making Platform}

In this paper, a visualization platform is developed for Co-operative Decision-Making in wind farm planning. This platform enables the major parties involved in the project to acquire an upfront understanding of how various co-related factors collectively affect the overall wind farm performance and local impact. Specifically, the wind farm performance in this work is represented by the wind farm Capacity Factor $(\mathrm{CF})$ and the Land Area per MW Installed (LAMI). Therefore, we perform a multi-objective (bi-objective) wind farm layout optimization to explore the CF-LAMI trade-off for different nameplate capacities. In addition, we also explore the variation of the land shapes associated with the best CF-LAMI trade-offs by applying an Optimal Layout-based (OL-based) land usage in the optimization. A GUI-based land shape chart estimation is finally developed to provide information regarding the interplay of the following planning elements: (i) $\mathrm{CF}$, (ii) LAMI, (iii) nameplate capacity, (iv) OL-based land shape, and (v) the average energy production potential. For example, the involved parties can now readily visualize how different LAMI and nameplate capacity decisions impact the estimated land portion/plots that need to be used and the maximum energy production that could be extracted under such decisions.

The formulation of the bi-objective optimization and the OL-based land usage will be presented in the following subsections. Descriptions of the visualization platform is presented in Section III.

\section{A. Optimization Problem Formulation}

There are two objectives in this paper: (i) maximizing the wind farm Capacity Factor, (ii) and minimizing the Land Area per MW Installed. However, our previous work has illustrated that a wind farm has large land area can produce more energy. ${ }^{15}$ Hence, it allows us to solve this bi-objective optimization problem as a constrained single-objective optimization problem. Besides, in this work we focus on large utility-scale wind farms. Considering the computational efficiency, a single objective optimizer is preferred. The constrained single-objective optimization problem is formulated as

$$
\begin{array}{ll} 
& \max C F(V) \\
& V=\left\{x_{1}, x_{2}, \cdots, x_{N}, y_{1}, y_{2}, \cdots, y_{N}\right\} \\
\text { subject to } & \\
& g_{1}(V) \geq 2 D \\
& g_{2}(V) \leq A_{M W}^{i}
\end{array}
$$

where $C F(V)$ represents the wind farm $\mathrm{CF}$ computed using Eq. 2; $V$ is the design variable that represents the location of turbines; $N$ is the total number of turbines installed; $D$ is the rotor diameter of the turbine used; and $g_{1}(V)$ represents the inner-turbine spacing, which given by

where

$$
g_{1}(V)=\sum \max \left\{\left(d_{i j}-2 D\right), 0\right\}
$$

$$
\begin{aligned}
& d_{i j}=\sqrt{\left(x_{i}-x_{j}\right)^{2}+\left(y_{i}-y_{j}\right)^{2}} \\
& i, j=1,2, \ldots, N, \text { and } i \neq j
\end{aligned}
$$

In Eq. 3, $g_{2}(V)$ represents the constrained land area based on the nameplate capacity (number of turbines), $A_{M W}^{i}$. By specifying multiple values of $A_{M W}^{i}$, the trade-off between CF and LAMI can be obtained and the comparison of CF for the same LAMI is also enabled. The Mixed-Discrete Particle Swarm Optimization (MDPSO) algorithm is used in this work to perform the layout optimization. For each given $A_{M W}^{i}$, the algorithm is run at least 5 times. The optimal solution (farm layout) is then selected as the best solution out of the 5 runs. Detailed information of the MDPSO algorithm can be found in the paper by Chowdhury et al. ${ }^{16}$ The Sobol's quasi-random sequence generator ${ }^{17}$ is applied to provide the initial set of farm layouts.

\section{B. The Optimal Layout-based Land Use}

In this paper, the land usage of a wind farm is determined by the farm layout or turbine arrangement. Hence, the Layout-based land use is derived from optimizing the location of turbines without specifying farm boundaries. For any given pattern of farm layout, the "2D convex hull" enclosing all turbines is determined. The Graham scan algorithm ${ }^{18}$ is applied to find turbines that comprise the facets of the $2 \mathrm{D}$ 


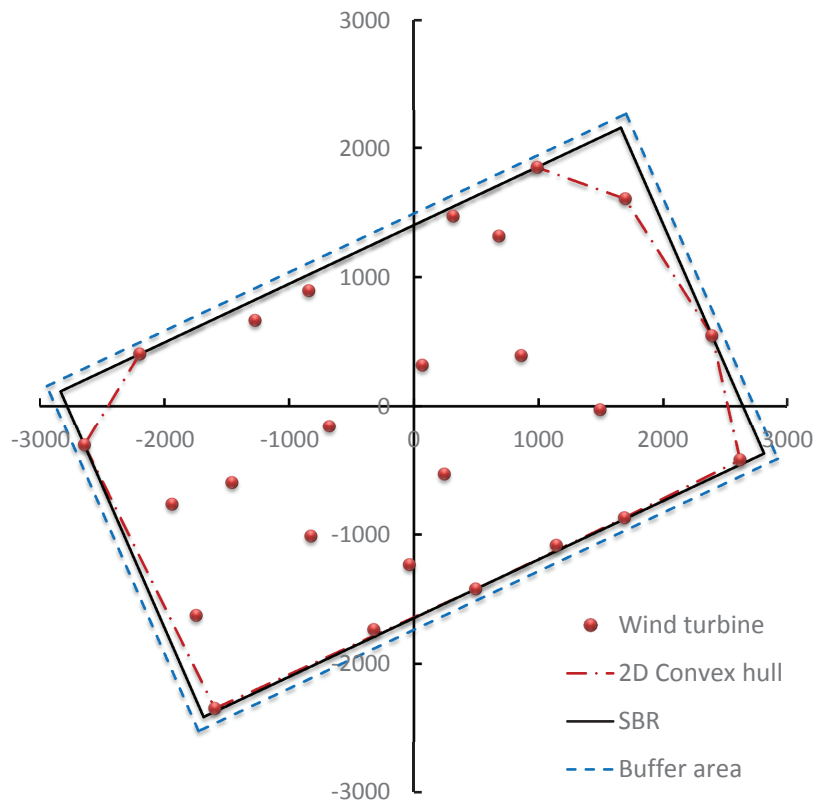

Figure 2. Illustration of the land use for a farm layout

convex hull. Considering wind farms generally have a rectangular shape, and that land is sold on leased as rectangular plots, the land shape in this paper is then determined by the Smallest Bounding Rectangle (SBR) enclosing all turbines, which is computed using the rotating calipers algorithm. ${ }^{19}$ A buffer zone, equivalent to 1 rotor diameter, is then added to each side to yield the final land use estimate.

Specifically, the land usage in this paper is determined based on the optimal wind farm layout (OLbased), which is evaluated within the process of wind farm layout optimization. As shown in Fig. 2, the region enclosed by the solid line box represents the SBR, and the OL-based land use ("including buffer area") is denoted by the dash line. Based on a known wind condition, this OL-based land use leads to the best utilization of the land resource (land area, land shape, and land orientation) for a given number of turbines. Moreover, the OL-based land use also allows the instant determination of the maximum farm output potential. Detailed information regarding the implementation of the OL-based land use can be found in the previous work by Tong et al. ${ }^{15}$

\section{Numerical Experiment}

In this Section, numerical experiments are conducted to generate the CF-LAMI trade-off and the GUIbased land shape chart, for a set of representative wind resource scenarios. Three different wind patterns (shown in Fig. 3) generated using the same wind distribution are used to allow a general appreciation of the benefits of such a wind farm layout optimization-based visualization platform.

Table 1. GE 1.5 MW xle Turbine ${ }^{20}$

\begin{tabular}{lc} 
Specifications & Value \\
\hline Rated capacity & $1500 \mathrm{~kW}$ \\
Cut-in & $3.5 \mathrm{~m} / \mathrm{s}$ \\
Cut-out & $20 \mathrm{~m} / \mathrm{s}$ \\
Rated speed & $11.5 \mathrm{~m} / \mathrm{s}$ \\
Rotor diameter & $82.50 \mathrm{~m}$ \\
Hub height & $80 \mathrm{~m}$
\end{tabular}




\section{A. Description and Settings}

In the wind diagrams in Fig. 3, the angular coordinate, showing the direction wind is flowing from, is measured clockwise from North. According to the wind patterns, three cases are considered in this paper:

Case 1: The wind pattern in Case 1 (as shown in Fig. 3(a)) comprises one dominant wind direction at an angle of $45^{\circ}$.

Case 2: The wind pattern in Case 2 comprises two dominant directions that are opposite to each other at angles of $45^{\circ}$ and $-135^{\circ}$, as shown in Fig. 3(b).

Case 3: The wind pattern in Case 3 also comprises two dominant directions, which are however orthogonal to each other at angles of $45^{\circ}$ and $-45^{\circ}$ as shown in Fig. 3(c).

These three patterns are generated from the same wind distribution fitted using simulated wind data, to ensure that they have equal wind power density (WPD).

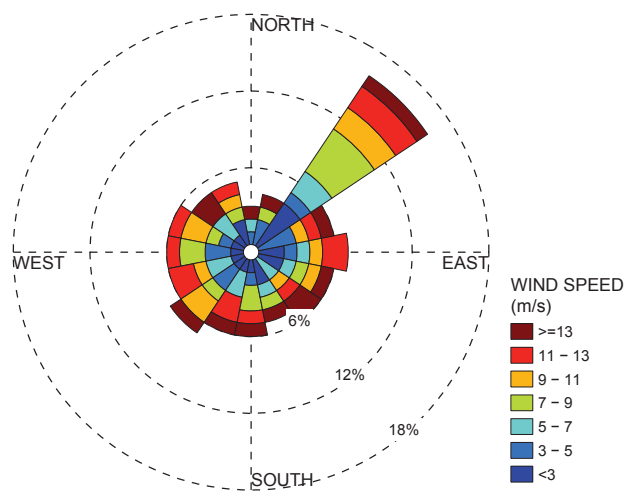

(a) Case 1: Single dominant direction

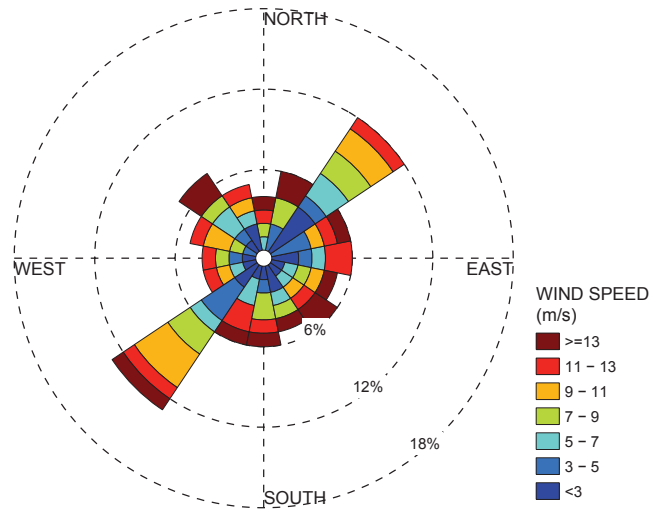

(b) Case 2: Two opposite dominant directions

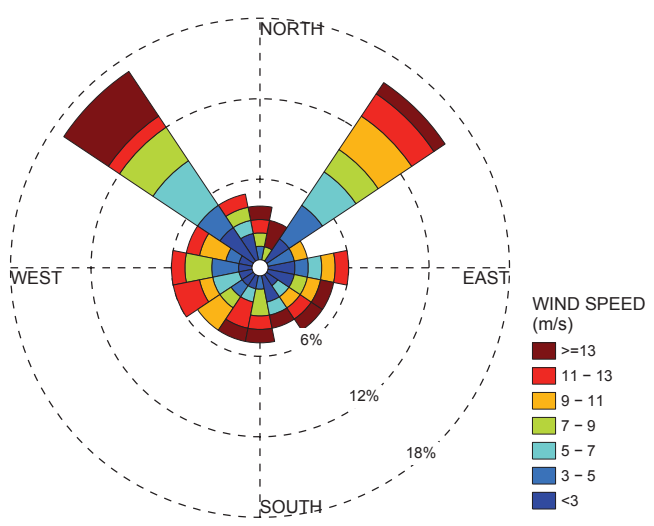

(c) Case 3: Two orthogonal dominant directions

Figure 3. Wind diagrams

For each wind pattern, 5 different values of nameplate capacities are also considered, which range from $37.5 \mathrm{MW}$ to $187.5 \mathrm{MW}$. The number of turbines is then ranged from 25 to 125 . Five different values of the LAMI are also selected, ranging from $40 h a / M W$ to $120 h a / M W$. Therefore, totally 75 wind farm optimizations are required in this experiment. For each case, optimization is run for 5 times to compensate for the impact of random parameters in the stochastic optimization used in this paper (which is MDPSO). These optimizations were carried out parallelly on 4 workstations, and this overall framework was (macrograined) parallelized to also fully exploit the multi cores $(4 / 8$ cores) architecture of each workstation. A GUI-based land shape chart is then generated for each wind pattern, illustrating the variation of OL-based land shape and maximized CF with different values of LAMI and nameplate capacity.

The major assumptions made in this numerical experiment are listed below: 
i. identical turbines (GE1.5 MW xle as shown in Table 1) are used;

ii. the incoming wind speed is uniformly distributed over the entire rotor area; and

iii. the ambient turbulence over the entire farm site is assumed constant.

\section{B. Results and Discussion}

Figure $4-6$ represent the land shape charts for the three wind patterns. This paper focuses on the impact of land use on wind farm performance, so the outline of each OL-based land shape is specifically shown as the smallest bounding rectangle. The outer x-axis and y-axis in the chart represent the LAMI and the number of turbines (nameplate capacity), respectively. The CF obtained from each of the optimal farm layouts are given by the color of the estimated rectangular wind farm bounding. The average AEP potential obtained by each optimal layout is shown at the center of the estimated rectangular farm bounding.

Figure 4 shows the results obtained in Case 1. Expectedly, for the same number of turbines installed (along each each row), the estimated CF improves as the land area increases; and for the same allowed LAMI (along each column), the estimated maximum CF reduces as the nameplate capacity increases (indicating more crowding of turbines). Therefore, throughout the entire chart the OL-based land shape located at the top-left corner yields the lowest CF; while the one at the bottom-right corner yields the highest CF. This trend is also observed in the other two land shape charts obtained in Case 2 and Case 3 (as shown in Fig. 5 and Fig. 6, respectively). This scenario is mainly attributed to the wake effect: for a given number of turbines installed, the greater the land area, the greater the inner-turbine spacing, thus leading to a reduction of wake losses. On the contrary, installing more turbines in a limited size of farm site will decrease the inner-turbine spacing, thereby causing more energy losses.

A more interesting observation is that, in Case 1, the OL-based land shapes for the lowest nameplate capacity (25 turbines, shown in the first row of the land shape chart shown in Fig. 4) experienced the most significant change in land aspect ratio; on the other hand, the most significant change in land orientation is experienced by the OL-based land shapes corresponding to the lowest allowed LAMI (40 ha/MW, in the first column of the land shape chart). A similar scenario is observed for the OL-based land shapes obtained in Case 2. However, in Case 3, the OL-based land shapes corresponding to either the lowest LAMI or the lowest nameplate capacity are experienced the most significant change in both the land orientation and aspect ratio. Overall, this initial investigation indicates that, for small allowed LAMI and for wind farm with few turbines, the OL-based land shape is highly sensitive to the variable factors (between LAMI and nameplate capacity).

It is also observed that, under the same planned nameplate capacity and LAMI, the OL-based land shapes obtained in Case 1 and Case 2 are relatively similar (as shown in Fig. 4 and Fig. 5); the only exception to this observation occurs in the case of the smallest allowed LAMI (which is $40 h a / M W$ ). Additionally, we observe that, under the same planned nameplate capacity and LAMI, the average AEP potential obtained in Case 1 and Case 2 are also very close to each other - the average estimated difference being $0.06 \%$ (the maximum AEP difference observed is around $1440 \mathrm{MWh} / \mathrm{yr}$ ). Put this observation into perspective, it should be noted that across the range of LAMI the average AEP potential varies by $4.37 \%$, and across the range of nameplate capacity the average AEP potential varies by $4.42 \%$. Nevertheless, from the land shape chart shown in Fig. 6, it is observed that the OL-based land shapes obtained in Case 3 are noticeably different from those obtained in Case 1 and Case 2 for the same nameplate capacity and LAMI. For the same nameplate capacity and LAMI, the different in the average AEP potential in Case 1 and Case 3 is equal to $0.47 \%$ on average (the maximum AEP differen observed is around $3610 \mathrm{MWh} / \mathrm{yr}$ ).

Based on the response surface of wind farm capacity factor developed by Chowdhury et al, ${ }^{9}$ it was concluded that, for a given farm site, a high capacity factor can be obtained with a relatively large land aspect ratio. However, we observed that some of the OL-based land shapes have a square shape (the land aspect ratio is very close to 1). This difference can be attributed to the wind distributions used in this paper, which essentially involve one or two clearly defined dominant wind directions, unlike the complex real data-based multi-modal wind distribution used by Chowdhury et alc̃iteSouma-JERT13. 


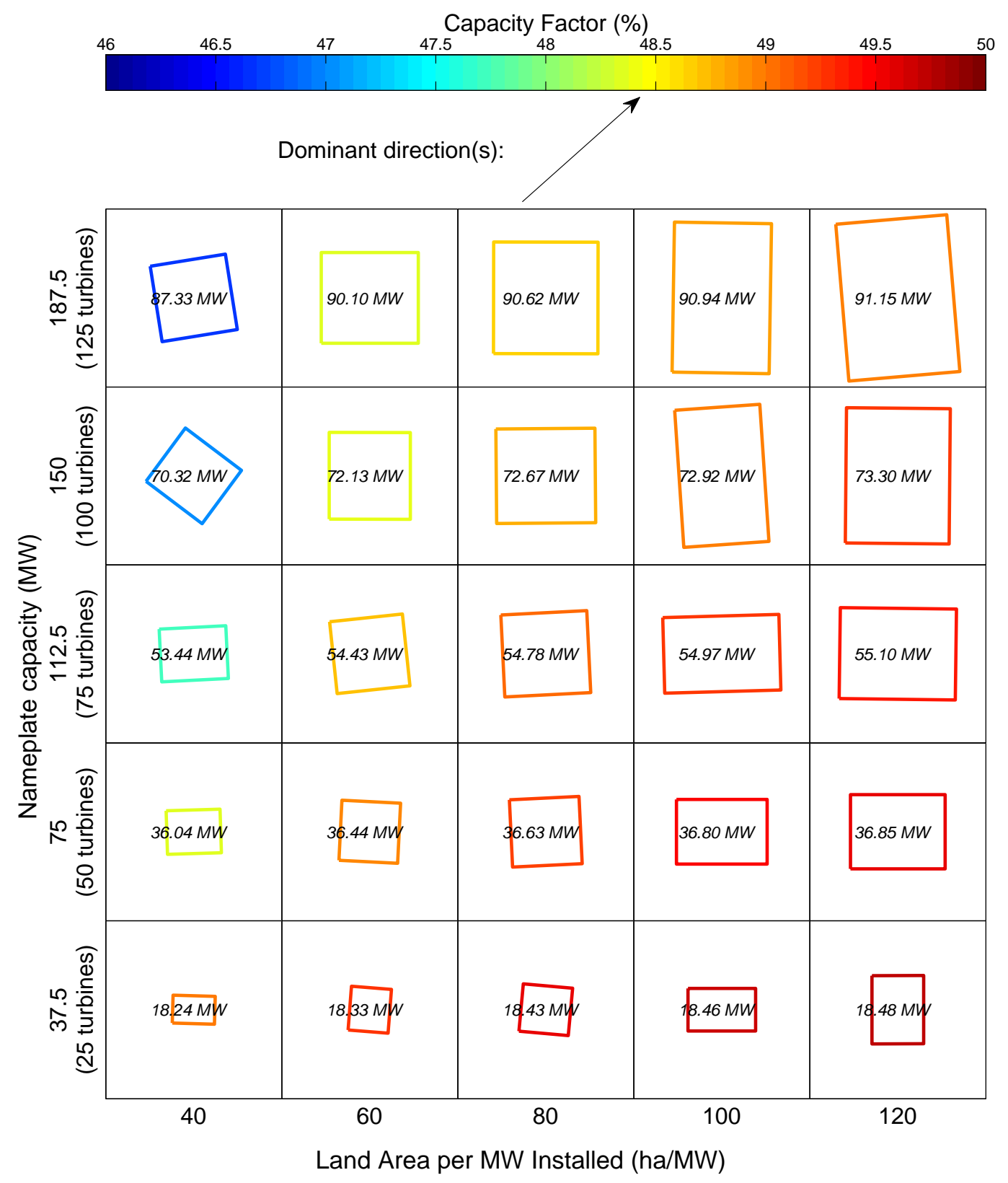

Figure 4. Case 1: GUI-based land shape chart under single dominant wind direction, with the average AEP potential $(M W)$ of each layout as shown at the center of each cell 


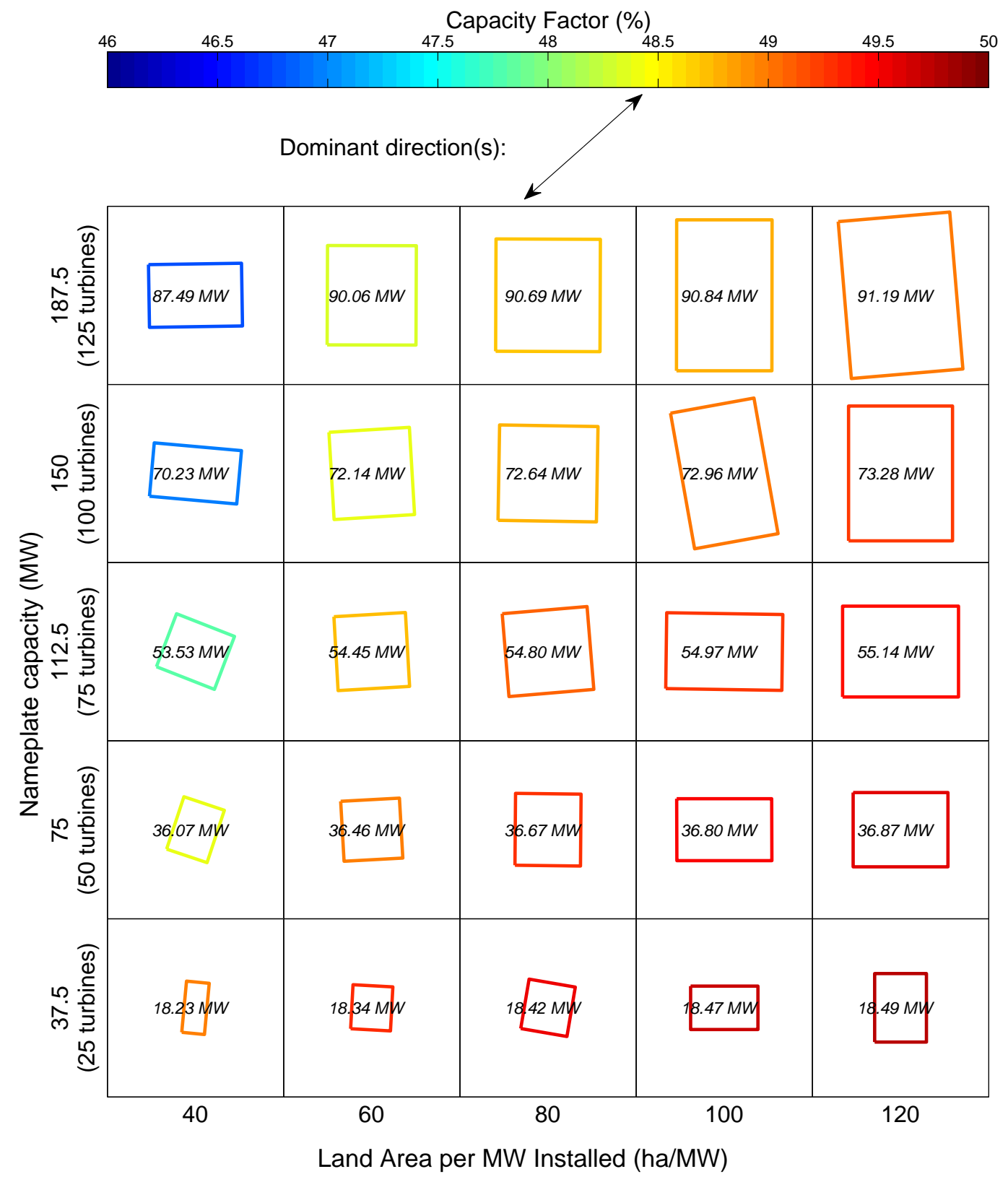

Figure 5. Case 2: GUI-based land shape chart under opposite dominant wind directions, with the average AEP potential $(M W)$ of each layout as shown at the center of each cell 

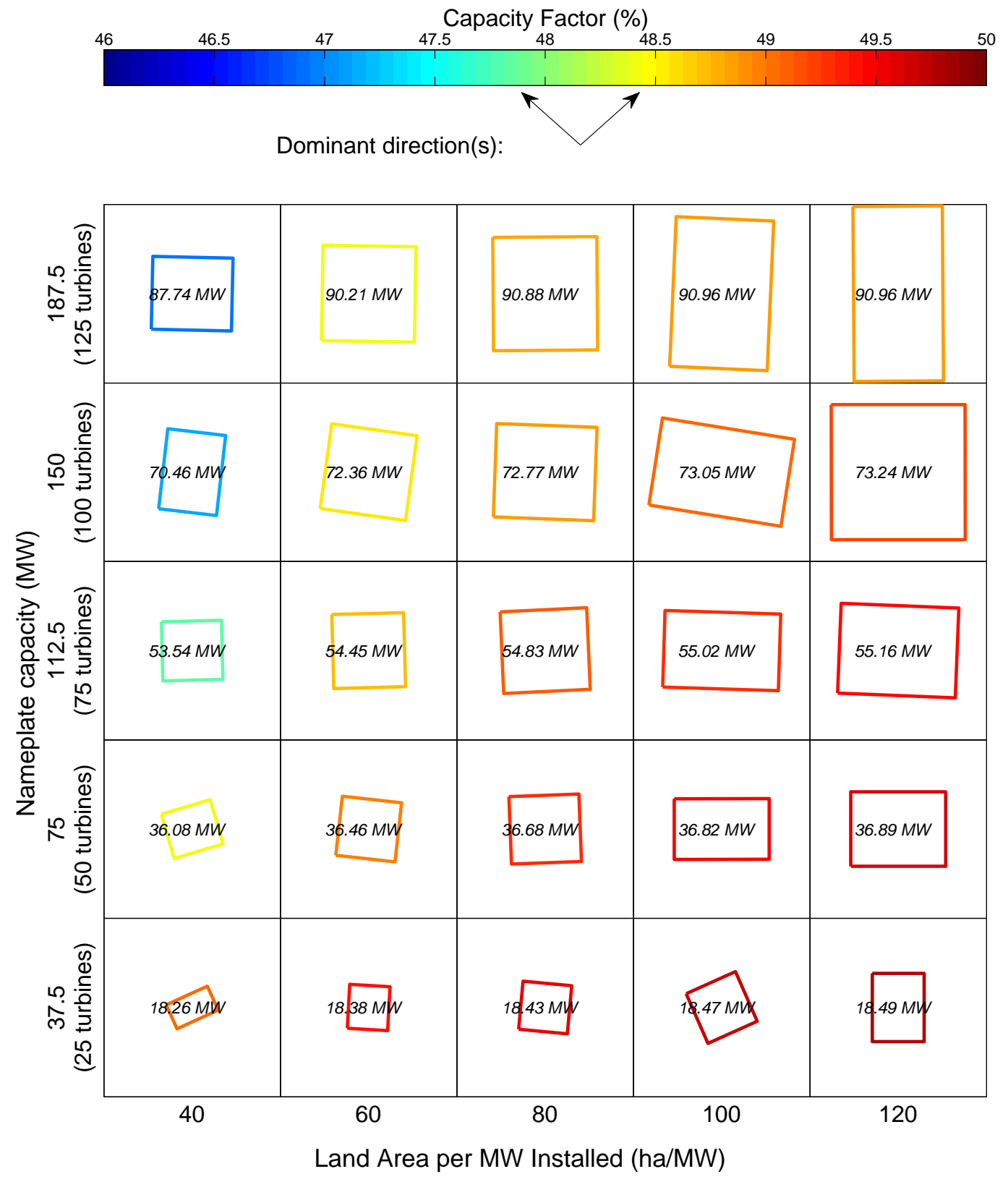

Figure 6. Case 3: GUI-based land shape chart under orthogonal dominant wind directions, with the average AEP potential $(M W)$ of each layout as shown at the center of each cell 


\section{Concluding Remarks}

This paper developed a trade-off visualization platform that is expected to promote Cooperative Decision Making platform. This platform is developed by performing multiple wind farm layout optimizations, to obtain the useful information guiding the wind farm planning process. Due to the nature of the defined problem and the consideration of computational efficiency, we solve the essentially bi-objective optimization problem as multiple constrained single objective optimization problems. The subsequent application of the Optimal Layout-based land use (obtained using 2D convex hull and the smallest bounding rectangle concepts) enabled the optimization to run without specifying farm boundaries. A novel GUI-based land shape chart is then developed to explore the CF-LAMI trade-off while also providing the OL-based land shapes and the average AEP potential for different values of nameplate capacities and LAMI.

The visualization platform was applied to three distinct wind patterns: (i) single dominant direction, (ii) two opposite dominant directions, and (iii) two orthogonal dominant directions; all these cases involved the same distribution of wind speed. The results represented by the GUI-based land shape charts showed that the optimal land shapes are self-oriented by the dominant wind direction(s). Moreover, the OL-based land shape is highly sensitive to the number of turbines in the case of small allowed LAMI and (vice versa) to the LAMI in the case of small installed capacity (few turbines installations). We also noted that, for a given number of turbines, the predicted CF improves as the LAMI increases; corollarily, the predicted CF decreases if more turbines are installed with the same LAMI. Additionally, under the same nameplate capacity and LAMI, the OL-based land shape obtained in Case 1 (with single dominant wind direction) and Case 2 (with two opposite dominant wind directions) is very similar; however, the OL-based land shape obtained in Case 1 and Case 3 (with two orthogonal dominant wind directions) is noticeably different. Across three cases, the difference in the estimated maximum AEP can be up to $3610 \mathrm{MWh} / \mathrm{yr}$.

Overall, the GUI-based land shape chart enables the integration of various key factors and objectives in wind farm planning, and hence provides insightful information to assist the major stakeholders in making favorable co-operative decisions.

Future work should enable the illustration of the actual degree of impact on surroundings (e.g., noise impact and impact on local wildlife) in the GUI-based land shape chart. In addition, the consideration of the land plot ownership and landowner participation, by overlaying this information (as a map) on the current chart, will lay the foundation for breakthrough advancements in wind farm planning.

\section{Acknowledgments}

Support from the National Science Foundation Awards CMMI-1100948, and CMMI-0946765 is gratefully acknowledged. Any opinions, findings, and conclusions or recommendations expressed in this paper are those of the authors and do not necessarily reflect the views of the NSF.

\section{References}

\footnotetext{
${ }^{1}$ Wayne Walker Conservation Consulting LLC, "An Overview of the Wind Power Project Development Process and Financial Performance of Wind Eerngy Projects," http://www.fws.gov/habitatconservation/windpower/past_meeting_ presentations/walker.pdf, February 2008, Accessed: November 2013.

${ }^{2}$ Siting Subcommittee of National Wind Coordinating Committee (NWCC), "Permitting of Wind Eerngy Facilities," http://www.nationalwind.org/assets/publications/permitting2002.pdf, August 2002.

${ }^{3}$ Mulvaney, K. K., Woodson, P., and Prokopy, L. S., "Different Shades of Green: A Case Study of Support for Wind Farms in the Rural Midwest," Environmental Management, Vol. 51, No. 5, May 2013, pp. 1012-1024.

${ }^{4}$ International Energy Agency (IEA), "IEA WInd RP14: Social Acceptance of Wind Energy Projects," Tech. rep., IEA, Paris, France, January 2013, Task 28.

${ }^{5}$ IEA, "Technology Roadmap: Wind Energy," Tech. rep., IEA, Paris, France, 2013.

${ }^{6}$ Aitken, M., "Wind Power Planning and Public Engagement: Challenges and Opportunities," Tech. Rep. 8, The Institue for the Study of Science Technology and Innovation (ISSTI), May 2010, ISSTI Briefing Note.

${ }^{7}$ Rynne, S., Flowers, L., Lantz, E., and Heller, E., "Planning for Wind Energy," Tech. Rep. 566, American Planning Associate (APA), Chicago, Illinois, 2013, Planning Advisory Service Report.

${ }^{8}$ Breukers, S. and Wolsink, M., "Wind power implementation in changing institutional landscapes," Energy Policy, Vol. 35, No. 5, 2007, pp. 2737-2750.

${ }^{9}$ Chowdhury, S., Zhang, J., Tong, W., and Messac, A., "Modeling the Influence of Land-Shape on the Energy Production Potential of a Wind Farm Site," Journal of Energy Resources Technology, (in press).
} 
${ }^{10}$ Rosenbloom, E., "A Problem With Wind Power," http://www.aweo.org/ProblemWithWind.pdf, September 2006, Accessed: November 2013.

${ }^{11}$ McEowen, R. A., "Wind energy production: Legal issues and related liability concerns for landowners," http://www.calt. iastate.edu/briefs/CALT $\backslash \% 20$ Legal $\backslash \% 20$ Brief $\backslash \% 20-\backslash \% 20$ Wind $\backslash \% 20$ Energy $\backslash \% 20$ Production.pdf, June 2011, Accessed: November 2013.

${ }^{12}$ Chowdhury, S., Zhang, J., Messac, A., and Castillo, L., "Characterizing The Influence of Land Configuration on The Optimal Wind Farm Performance," ASME 2011 International Design Engineering Technical Conferences (IDETC), ASME, Washington, DC, August 28-31 2011.

${ }^{13}$ Chowdhury, S., Zhang, J., Messac, A., and Castillo, L., "Characterizing The Influence of Land Area and Nameplate Capacity on The Optimal Wind Farm Performance," ASME 2012 6th International Conference on Energy Sustainability, ASME, San Diego, July 23-26 2012.

${ }^{14}$ Chowdhury, S., Zhang, J., Messac, A., and Castillo, L., "Optimizing the arrangement and the selection of turbines for wind farms subject to varying wind conditions," Renewable Energy, Vol. 52, April 2013, pp. 273-282.

${ }^{15}$ Tong, W., Chowdhury, S., Mehmani, A., and Messac, A., "Multi-objective Wind Farm Design: Exploring the Trade-off between Capacity Factor and Land Use," 10th World Congress on Structural and Multidisciplinary Optimization (WCSMO), ISSMO, Orlando, Florida, USA, May 19-24 2013.

${ }^{16}$ Chowdhury, S., Tong, W., Messac, A., and Zhang, J., "A Mixed-Discrete Particle Swarm Optimization Algorithm with Explicit Diversity-Preservation," Structural and Multidisciplinary Optimization, Vol. 47, 2013, pp. 367-388.

${ }^{17}$ Sobol, I. M., "On the Distribution of Points in a Cube and the Approximate Evaluation of Integrals," Zh. Vychisl. Mat. Mat. Fiz., Vol. 7, No. 4, 1967, pp. 784-802.

${ }^{18}$ Graham, R., "An Efficient Algorithm for Determining the Convex Hull of a Finite Planar Set," Information Processing Letters, Vol. 1, 1972, pp. 132-133. 1983.

${ }^{19}$ Toussaint, G., "Solving Geometric Problems with the Rotating Calipers," Proc. MELECON '83, IEEE, Athens, Greece,

${ }^{20} \mathrm{GE}$ Energy, "1.5MW Wind Turbine," http://geosci.uchicago.edu/ moyer/GE0S24705/Readings/

